\title{
Effect of hyperglycaemia on inflammatory and stress responses and clinical outcome of pneumonia in non-critical-care inpatients: results from an observational cohort study
}

\author{
Philipp Schuetz • Natalie Friedli • Eva Grolimund • Alexander Kutz • Sebastian Haubitz • \\ Mirjam Christ-Crain • Robert Thomann • Werner Zimmerli • Claus Hoess • \\ Christoph Henzen • Beat Mueller • for the ProHOSP Study Group
}

Received: 6 July 2013 / Accepted: 31 October 2013 /Published online: 24 November 2013

(C) Springer-Verlag Berlin Heidelberg 2013

\begin{abstract}
Aims/hypothesis Despite the condition's high prevalence, the influence of hyperglycaemia on clinical outcomes in noncritical-care inpatients with infections remains ill defined. In this study, we analysed associations of glucose levels at admission and during initial inpatient treatment with the
\end{abstract}

Philipp Schuetz and Natalie Friedli contributed equally to this work

Electronic supplementary material The online version of this article (doi:10.1007/s00125-013-3112-9) contains peer-reviewed but unedited supplementary material, which is available to authorised users.

P. Schuetz $(\bowtie) \cdot$ N. Friedli $\cdot$ E. Grolimund $\cdot$ A. Kutz $\cdot$ S. Haubitz $・$ B. Mueller

University Department of Internal Medicine, Kantonsspital Aarau, Tellstrasse, 5001 Aarau, Switzerland

e-mail: schuetzph@gmail.com

\section{Christ-Crain}

Department of Internal Medicine, Division of Endocrinology, Diabetes and Clinical Nutrition, University Hospital Basel, Basel, Switzerland

R. Thomann

Department of Internal Medicine, Buergerspital Solothurn,

Solothurn, Switzerland

W. Zimmerli

Department of Internal Medicine, Kantonsspital Liestal, Liestal, Switzerland

C. Hoess

Department of Internal Medicine, Kantonsspital Münsterlingen, Münsterlingen, Switzerland

C. Henzen

Department of Internal Medicine, Kantonsspital Lucerne, Lucerne, Switzerland inflammatory response and clinical outcome in communityacquired pneumonia (CAP) patients.

Methods This secondary observational analysis included 880 confirmed CAP patients. We used severity-adjusted multivariate regression models to investigate associations of initial and $96 \mathrm{~h}$ mean glucose levels with serially measured biomarker levels over 7 days (C-reactive protein [CRP], procalcitonin, white blood cell count [WBC], pro-adrenomedullin [ProADM]) and adverse clinical course (death and intensivecare unit admission).

Results In the 724 non-diabetic patients $(82.3 \%$ of the study population), moderate or severe hyperglycaemia (glucose 6-11 $\mathrm{mmol} / \mathrm{l}$ and $>11 \mathrm{mmol} / \mathrm{l}$, respectively) was associated with increased risk for adverse clinical course (adjusted OR [95\% CI] $1.4[0.8,2.4]$ and $3.0[1.1,8.0]$, respectively) and with higher CRP, WBC and ProADM levels over 7 days $(p<0.05$, ANOVA, all days). In diabetic patients $(n=156)$, no similar associations were found for initial hyperglycaemia, although mean $96 \mathrm{~h}$ glucose levels $\geq 9 \mathrm{mmol} / \mathrm{l}$ were associated with adverse clinical course (adjusted OR 5.4 [1.1, 25.8]; $p=0.03$ ). No effect modification by insulin treatment was detected (interaction terms $p>0.2$ for all analyses).

Conclusions/interpretation Initial hyperglycaemia in nondiabetic CAP patients, and prolonged hyperglycaemia in diabetic or non-diabetic CAP patients, are associated with a more pronounced inflammatory response and CAP-related adverse clinical outcome. Optimal glucose targets for insulin treatment of hyperglycaemia in non-critical-care settings should be defined.

Keywords Biomarker response - Diabetes · Glucose · Hyperglycaemia $\cdot$ Outcome $\cdot$ Pneumonia 


\begin{tabular}{|c|c|}
\hline \multicolumn{2}{|c|}{ Abbreviations } \\
\hline CAP & Community-acquired pneumonia \\
\hline CRP & C-reactive protein \\
\hline CURB65 & $\begin{array}{l}\text { Confusion, blood urea nitrogen, respiratory rate, } \\
\text { blood pressure, age } \geq 65 \text { years }\end{array}$ \\
\hline ED & Emergency department \\
\hline ICU & Intensive-care unit \\
\hline PCT & Procalcitonin \\
\hline ProADM & Pro-adrenomedullin \\
\hline ProHOSP & $\begin{array}{l}\text { Procalcitonin Guided Antibiotic Therapy and } \\
\text { Hospitalisation in Patients with Lower Respira- } \\
\text { tory Tract Infections }\end{array}$ \\
\hline WBC & White blood cell count \\
\hline
\end{tabular}

\section{Introduction}

Preclinical data suggest that hyperglycaemia has a profound influence on immune function. Hyperglycaemia interacts with components of the innate immune system in vitro, such as chemotaxis, phagocytosis and macrophage activation [1-3]. It also interacts with the adaptive immune system and directly inhibits T-lymphocyte, immunoglobulin and complement functions $[4,5]$. In animal models of diabetes, hyperglycaemia was associated with decreased bacterial clearance and higher infection-related mortality [6].

Yet, results from studies of clinical outcomes in hyperglycaemic patients with severe bacterial infections outside critical care remain ambiguous. Some studies reported higher mortality rates among those with hyperglycaemia, while others found no influence of glucose homeostasis on outcomes (reviewed in [3]). For patients with community-acquired pneumonia (CAP), a recent large observational study found a stepwise increase in mortality with higher initial blood glucose levels [7]. Interestingly, certain investigators reported a protective effect of diabetes when examining infection outcomes [8-11]. Although the underlying mechanisms have not been well understood, it has been speculated that diabetic individuals with chronic hyperglycaemia are protected from toxic effects exerted by acute (stress) hyperglycaemia [3]. Trials regarding the prevention and treatment of hyperglycaemia have investigated the effects of tight glycaemic control on patient outcomes. While initial trials favoured tight, near euglycaemic, glucose control, more recent research found that prevention of hypoglycaemia and high glucose variability with more supraphysiological glucose target ranges (i.e. $6-10 \mathrm{mmol} / \mathrm{l}$ ) ultimately may be the preferred strategy [12-14].

Importantly, the latter trials, as well as most research into the influence of glycaemia on the clinical outcome of bacterial infection, have been conducted in the critical-care setting, where insulin is given via infusion and patients are closely monitored. Few published studies have focused on inpatients with systemic infections in the non-critical-care setting, where insulin is given subcutaneously and is less closely monitored. The influence of hyperglycaemia on clinical outcomes in this latter population therefore remains ill defined. A recent large study including patients with sepsis of varying infectious aetiology hospitalised in a non-critical-care ward found that higher glucose concentrations within the first 72 inpatient hours were associated with worse hospital outcomes in the nondiabetic population [15]. Although this association remained significant after adjustment for severity of illness, residual confounding could not be excluded. Interestingly, diabetic patients did not show the same association, which led us to hypothesise that because of chronic hyperglycaemic states, diabetic patients may have an altered response to glucose.

We therefore performed a secondary analysis of a completed prospective multicentre observational trial $[16,17]$ to study the association of initial or $96 \mathrm{~h}$ mean glucose levels with inflammatory and stress responses - reflected by serial measurement of four blood biomarkers - as well as with clinical outcomes in a large, well-characterised cohort of diabetic and non-diabetic patients with CAP.

\section{Methods}

Setting and study design This observational analysis included all patients with radiologically confirmed CAP participating in an investigator-initiated, prospective, randomised, controlled, multicentre antibiotic stewardship trial (Procalcitonin Guided Antibiotic Therapy and Hospitalisation in Patients With Lower Respiratory Tract Infections [ProHOSP] study) [17]. Details of the study design were published previously [16]. Briefly, of 1,825 screened patients with respiratory symptoms, this trial enrolled 1,359 consecutive patients with presumed lower respiratory tract infection presenting to emergency departments (EDs) in any of six tertiary care hospitals in Switzerland. A total of 237 patients were ineligible (included during earlier hospitalisations, not fluent in German, refused participation) and a total of 207 patients were excluded because of severe immunosuppression $(n=51)$, concomitant infection $(n=29)$, active drug abuse $(n=25)$, hospital-acquired pneumonia $(n=45)$, expected imminently terminal comorbidity $(n=45)$ or other reasons $(n=12)$. In all, 925 patients had a final diagnosis of CAP with an infiltrate in the initial chest X-ray, of whom 880 (95.1\%) had an initial glucose measurement at ED admission and were thus eligible for this analysis.

The trial's main objective was to assess the effect of procalcitonin (PCT) monitoring on antibiotic consumption during the hospital stay and on clinical outcomes during the 30 days post admission. Patients were randomised 1:1 to use or non-use of serial PCT measurements as an input into the attending physicians' decision making regarding whether to start and how long to continue antibiotic therapy. Otherwise, patients with CAP in both study arms similarly received treatment for 
pneumonia according to state-of-the-art clinical guidelines and local best practice. In both groups, hypoglycaemia was treated based on local insulin protocols. Oral glucose-lowering treatment was stopped and insulin treatment, mostly sliding-scale insulin with or without basal insulin, was started to keep glucose in the near-normal range (i.e. $5-10 \mathrm{mmol} / \mathrm{l}$ ).

The study protocol was approved by all local ethical committees and written informed consent was obtained to collect blood on admission and during follow-up to measure biomarkers [17]. An independent monitoring committee assessed safety and adverse events during the trial. The study was registered in the 'Current Controlled Trials Database' (www.controlled-trials.com/ISRCTN95122877; identifier ISRCTN 95122877).

Hypothesis and general approach of this secondary analysis We hypothesised that hyperglycaemia on admission and during inpatient treatment would be associated with a more severe (i.e. stressful) infectious course of CAP, as demonstrated by a more pronounced inflammatory and stress response and worse clinical outcome. To test this hypothesis, we first investigated the association of initial blood glucose concentrations with blood biomarker levels over 7 days, as well as with 30 day clinical outcomes. Based on the initial glucose concentration, patients were a priori classified as having normal levels (reference group, blood glucose $<6 \mathrm{mmol} / \mathrm{l}$ ), moderate hyperglycaemia (blood glucose 6-11 mmol/1) or severe hyperglycaemia (blood glucose $>11 \mathrm{mmol} / \mathrm{l}$ ), based on the classification in a recent publication [7]. We used blood levels of C-reactive protein (CRP), PCT, white blood cell count (WBC) and pro-adrenomedullin (ProADM) from the day of admission and days 3,5 and 7 to reflect the inflammatory response. These markers are upregulated during systemic infections and correlate with inflammation (CRP, WBC), bacterial aetiology (PCT) and disease severity/mortality and other adverse outcome risk (ProADM) [18-22]. For the purpose of this study, we defined 'adverse clinical course' as all-cause mortality, intensive-care unit (ICU) admission, or both, during a 30 day follow-up.

In a second step, we assessed the association of mean glucose levels over the first $96 \mathrm{~h}$ of the hospital stay with biomarker levels and clinical outcomes as defined above. For this latter analysis, we focused on patients treated in the noncritical-care hospital ward with increased initial glucose levels $(>7.5 \mathrm{mmol} / \mathrm{l})(n=161)$. Due to this elevation, all such patients had repeated preprandial glucose measurements over the hospital course. Based on the mean glucose levels over 96 h, we classified patients as having normal glucose levels (reference group, blood glucose $<9 \mathrm{mmol} / \mathrm{l}$ ) or hyperglycaemic levels (blood glucose $\geq 9 \mathrm{mmol} / \mathrm{l}$ ). This a priori classification corresponds to guidelines by the Endocrine Society recommending that in-hospital glucose values outside critical care should be maintained in the range of $7.8-10 \mathrm{mmol} / 1$ [23].
Selection of participants, data collection and processing Patients eligible for this analysis were aged 18 years or older and were admitted from the community or a nursing home with CAP of less than 28 days' duration. CAP was defined by the presence of at least one respiratory symptom plus at least one finding during auscultation, or one sign of infection independent of antibiotic pretreatment, and a new infiltrate on chest radiograph [24-27]. Exclusion criteria were applied: inability to give written informed consent because of language restriction or severe dementia; active intravenous drug use; severe immunosuppression other than corticosteroid use; imminently life-threatening medical comorbidities; patients with hospital-acquired pneumonia (pneumonia onset $>48 \mathrm{~h}$ after the study hospital admission or hospitalisation within 14 days before ED presentation in ProHOSP) and patients with chronic infections necessitating long-term antibiotic treatment.

We classified patients as having pre-existing diabetes based on self-report or medical record documentation, or both. We recorded initial blood glucose values in the ED in all patients. We also recorded subsequent preprandial glucose measurements during the first $96 \mathrm{~h}$ of the hospital stay. For the time course, we calculated the mean glucose concentration (mean glucose) assuming a linear trend between measurements and using all available measured preprandial blood glucose concentrations within the first $96 \mathrm{~h}$.

Statistical analysis To describe the populations, values are expressed as means with SDs and frequencies as percentages or counts, as appropriate. We investigated the association of admission glucose and $96 \mathrm{~h}$ mean glucose values and outcomes in patients with or without diabetes. We stratified our analysis by presence of diabetes because previous studies suggested a differential impact of glycaemic states on clinical outcomes of severe bacterial infection in patients with or without that diagnosis. Admission glucose levels were analysed as categorical variables based on the following blood glucose ranges: $<6 \mathrm{mmol} / \mathrm{l}$ (reference group), 6-11 $\mathrm{mmol} / 1$ (mild hyperglycaemia) and $>11 \mathrm{mmol} / \mathrm{l}$ (severe hyperglycaemia). These cut-offs were predefined based on a recent large CAP study [7]. For mean 96 h glucose levels, we used only one cut-off (i.e. $9 \mathrm{mmol} / \mathrm{l}$, the median level) due to the low number of patients included. To assess whether insulin treatment interacts with the association of blood glucose and outcome (effect modification), we also included interaction terms in the statistical models.

The inflammatory response was graphically demonstrated by mean blood levels of four predefined biomarkers on the day of admission, and on days 3, 5 and 7. Association of glucose ranges and adverse outcomes was analysed using logistical regression with report of ORs and 95\% CIs. We adjusted the analysis for CAP severity at presentation using the initial confusion, blood urea nitrogen, respiratory rate, blood pressure, age $\geq 65$ years (CURB65) score, which is 
well-validated for such classification [28]. All analyses were performed with Stata12 (StataCorp, College Station, USA).

\section{Results}

Baseline characteristics Of 880 patients with a definitive diagnosis of CAP and initial blood glucose measurements, $156(17.7 \%)$ had a history of diabetes according to self-report, medical records, or both, overwhelmingly type 2 (98.1\%). Baseline characteristics of the study cohort according to diabetes history are presented in Table 1. Compared with nondiabetic patients, diabetic patients were older, had more frequent cardiovascular comorbidities, a higher BMI and higher mean $( \pm \mathrm{SD})$ glucose levels at admission $(11.1 \pm 5.3 \mathrm{mmol} / \mathrm{l} \mathrm{vs}$ $7.1 \pm 2.0 \mathrm{mmol} / \mathrm{l}, p<0.0001)$. On admission, the markers of inflammation/infection CRP, WBC and PCT were similar in diabetic and non-diabetic patients and ProADM was significantly higher in the diabetic population.

Association of initial glucose category with outcome In total, 112 patients $(12.7 \%, 95 \%$ CI $11.9 \%, 17.8 \%)$ had a complicated course, including death $(n=46)$, ICU admission $(n=80)$, or both ( $n=14)$, during the 30 day follow-up. Overall, there was no difference in mortality or adverse clinical course between diabetic and non-diabetic patients (respectively, $5.7 \%$ vs $5.1 \%$, $p=0.74$ and $16.3 \%$ vs $12.0 \%, p=0.17$ ) (Table 1 ).

In non-diabetic patients, there was a stepwise increase in the adverse clinical course rate in patients with moderate or severe admission hyperglycaemia compared with the reference group (Fig. 1a). This pattern was also true for the endpoints of ICU admission or mortality (Table 2). Logistical regression confirmed the increased risk associated with severe admission hyperglycaemia, with respective ORs of 4.0, 4.4 and 4.1 for ICU admission, mortality and the combined endpoint of adverse clinical course within 30 days in non-diabetic patients. These associations also held true in a regression analysis adjusted for initial CAP severity using the CURB65 score, with respective ORs of 3.0, 2.9 and 3.0.

In contrast, in diabetic patients risks were similar in the different admission glucose range groups and logistical regression analysis did not show significant associations for any of mortality, ICU admission or adverse clinical course (Table 2).

Association of initial glucose category with course of biomarker levels Figure 2 shows levels of the inflammation/ infection biomarkers WBC, CRP, PCT and ProADM on admission and on days 3, 5 and 7. In non-diabetic patients, higher admission glucose ranges were associated with higher initial and follow-up CRP, WBC and ProADM levels for all days $(p<0.05$, ANOVA), but not with PCT levels at these measurement points ( $p \geq 0.05$, ANOVA). In the diabetic subgroup, no significant differences were found, except for CRP on the day of admission $(p<0.05)$.

Association of $96 \mathrm{~h}$ mean glucose levels with outcome and inflammatory response We also investigated the association of mean blood glucose levels over the first 96 inpatient hours with clinical outcomes as well as biomarker levels. Patients had preprandial glucose measurements taken routinely with a mean $( \pm \mathrm{SD})$ of $2.8( \pm 0.80), 2.7( \pm 1.2), 2.5( \pm 1.3)$ and 2.2 $( \pm 1.4)$ measurements taken, respectively, on days 1, 2, 3 and 4 . A total of $70 \%$ of these patients were treated with subcutaneous sliding-scale insulin regimens, with a mean $( \pm \mathrm{SD})$ dose of $21( \pm 22), 22( \pm 23), 22( \pm 24)$ and $23( \pm 23) \mathrm{U}$, respectively, on days $1,2,3$ and 4 .

The risk for adverse outcome increased with increasing glucose levels, which, however, was more pronounced in the diabetic patient population $(p=0.015)$ and non-significant $(p=0.204)$ in the non-diabetic population (Fig. 1c, d). These observations held true in logistical regression analysis, where a mean glucose level of $\geq 9 \mathrm{mmol} / \mathrm{l}$ during the first $96 \mathrm{~h}$ of hospitalisation was found to be associated with increased risk of adverse clinical course in diabetic patients (adjusted OR 5.4, 95\% CI 1.1, 25.8; $p=0.03$ ) (Table 3). Again, this association was non-significant in patients without diabetes (adjusted OR 1.8, 95\% CI 0.4, 7.2; $p=0.43$ ). We did not find evidence for effect modification by insulin treatment $(p>0.2$ for interaction in diabetic and non-diabetic patients).

Figure 3 shows the kinetics of the four blood biomarkers in patients stratified by mean $96 \mathrm{~h}$ glucose range $(\leq 9.0 \mathrm{vs}$ $\geq 9.0 \mathrm{mmol} / \mathrm{l})$ and diabetes status. In diabetic patients, significant differences were found for CRP, with consistently higher levels in hyperglycaemic patients $(p<0.05$, ANOVA, for all measurements), and for WBC on days 5 and 7 ( $p<0.05$, ANOVA, for both measurements). For non-diabetic patients, no significant differences were found $(p>0.05$, ANOVA). Again, there was no evidence for effect modification of insulin treatment on the association of glucose range and biomarker level on any of the days $(p>0.2$ for all interactions in diabetic and non-diabetic individuals).

\section{Discussion}

The key findings of this study are threefold. First, in nondiabetic patients with CAP in the non-critical-care setting, initial hyperglycaemia was associated with a more pronounced inflammatory response as demonstrated by the evolution of biomarker levels over 7 days and adverse 30 day outcomes. These findings were independent of severity of illness, arguing against an exclusive stress-dependent hyperglycaemia, and in favour of the potential toxic effect of glucose elevation. Second, the same association of admission hyperglycaemia and outcome was not found in the diabetic 
Table 1 Patient characteristics by diabetes status

\begin{tabular}{|c|c|c|c|c|}
\hline by diabetes status & Characteristic & $\begin{array}{l}\text { Non-diabetic } \\
\text { patients }(n=724)\end{array}$ & $\begin{array}{l}\text { Diabetic } \\
\text { patients }(n=156)\end{array}$ & $p$ value \\
\hline & \multicolumn{4}{|l|}{ Demographic characteristics } \\
\hline & Age, years, mean $\pm \mathrm{SD}$ & $67 \pm 19.1$ & $74 \pm 10.4$ & $<0.0001$ \\
\hline & Male sex, \% $(n)$ & $57.2(414)$ & $66.7(104)$ & 0.029 \\
\hline & \multicolumn{4}{|l|}{ Serum glucose level on admission, mean $\pm \mathrm{SD}$} \\
\hline & Initial mean glucose level, mmol/l & $7.1 \pm 2.0$ & $11.1 \pm 5.3$ & $<0.0001$ \\
\hline & Initial glucose range, $\%(n)$ & & & $<0.001$ \\
\hline & Euglycaemia, $<6.0 \mathrm{mmol} / 1$ & $27.6(200)$ & $10.3(16)$ & \\
\hline & Moderate hyperglycaemia, 6-11 mmol/1 & $68.4(495)$ & $48.7(76)$ & \\
\hline & Severe hyperglycaemia, $>11 \mathrm{mmol} / 1$ & $4.0(29)$ & $41.0(64)$ & \\
\hline & \multicolumn{4}{|l|}{ Coexisting illnesses, $\%(n)$} \\
\hline & Chronic heart failure & $16.3(118)$ & $20.5(32)$ & 0.204 \\
\hline & Coronary arterial disease & $17.3(125)$ & $33.3(52)$ & $<0.0001$ \\
\hline & Cerebrovascular disease & $8.4(61)$ & $11.5(18)$ & 0.217 \\
\hline & Chronic renal failure & $18.1(131)$ & $40.4(63)$ & $<0.0001$ \\
\hline & Peripheral arterial disease & $4.3(31)$ & $9.0(14)$ & 0.016 \\
\hline & \multicolumn{4}{|l|}{ Cardiovascular risk factors } \\
\hline & Current or former nicotine use, $\%(n)^{\mathrm{a}}$ & $27.5(193)$ & $17.8(27)$ & 0.013 \\
\hline & Pack years, mean $\pm \mathrm{SD}$ & $38.6 \pm 27.6$ & $42.8 \pm 30.5$ & 0.3193 \\
\hline & $\mathrm{BMI}, \mathrm{kg} / \mathrm{m}^{2}$, mean $\pm \mathrm{SD}$ & $25.2 \pm 5.1$ & $28.5 \pm 5.9$ & $<0.0001$ \\
\hline & $\mathrm{BMI}>25 \mathrm{~kg} / \mathrm{m}^{2}, \%(n)$ & $56.2(407)$ & $76.3(119)$ & $<0.0001$ \\
\hline & \multicolumn{4}{|l|}{ Clinical history, $\%(n)^{\mathrm{a}}$} \\
\hline & Antibiotic pretreatment on presentation & $26.3(189)$ & $19.4(30)$ & 0.072 \\
\hline & Cough & $86.2(598)$ & $82.8(125)$ & 0.283 \\
\hline & Dyspnoea & $77.9(538)$ & $83.0(122)$ & 0.167 \\
\hline & Fever or chills & $68.6(493)$ & $60.7(94)$ & 0.057 \\
\hline & \multicolumn{4}{|l|}{ Clinical findings } \\
\hline & Systolic blood pressure, $\mathrm{mmHg}$, mean $\pm \mathrm{SD}$ & $133 \pm 22.8$ & $135 \pm 24.4$ & 0.292 \\
\hline & Diastolic blood pressure, $\mathrm{mmHg}$, mean $\pm \mathrm{SD}$ & $74 \pm 14.8$ & $73 \pm 14.5$ & 0.3116 \\
\hline & Heart rate, beats/min, mean $\pm \mathrm{SD}$ & $96 \pm 19.3$ & $96 \pm 18.5$ & 0.9638 \\
\hline & \multicolumn{4}{|l|}{ Treatment site, $\%(n)$} \\
\hline & Inpatient & $90.1(652)$ & $96.2(150)$ & 0.015 \\
\hline & \multicolumn{4}{|l|}{30 day outcome, $\%(n)$} \\
\hline & Adverse clinical course: all-cause death + ICU admission ${ }^{a}$ & $12.0(87)$ & $16.3(25)$ & 0.173 \\
\hline & All-cause death ${ }^{\mathrm{a}}$ & $5.1(37)$ & $5.7(9)$ & 0.737 \\
\hline & ICU admission & $8.4(61)$ & $12.2(19)$ & 0.139 \\
\hline & \multicolumn{4}{|l|}{ Initial laboratory findings, mean $\pm \mathrm{SD}$} \\
\hline & $\mathrm{PCT}, \mu \mathrm{g} / 1$ & $4.3 \pm 15.3$ & $4.2 \pm 8.2$ & 0.9462 \\
\hline & CRP, nmol/1 & $1,714 \pm 1.247$ & $1,647 \pm 1,190$ & 0.5609 \\
\hline & WBC, $10^{9} / 1$ & $13.6 \pm 7.2$ & $13.8 \pm 8.8$ & 0.8293 \\
\hline${ }^{\text {a }}$ Data not available for some & ProADM, nmol/1 & $1.5 \pm 1.5$ & $2.1 \pm 1.6$ & $<0.001$ \\
\hline
\end{tabular}

${ }^{\text {a }}$ Data not available for some patients population. Third, persistent hyperglycaemia over the first $96 \mathrm{~h}$ of inpatient treatment was associated with adverse clinical outcome and a more pronounced CRP response mainly in the diabetic population and independent of severity of illness.

Optimal glucose targets in the non-critical-care hospital setting have not been well defined. Still, guidelines of the
Endocrine Society and other organisations recommend maintaining in-hospital glucose values in the range of 7.8 $10 \mathrm{mmol} / \mathrm{l}$ [23]. This recommendation is mainly based on several large studies, principally from the critical-care setting, reporting associations of elevated initial blood glucose levels and increased adverse outcome risk in postoperative patients and patients with sepsis [3, 15, 29-32]. Aligned with these 

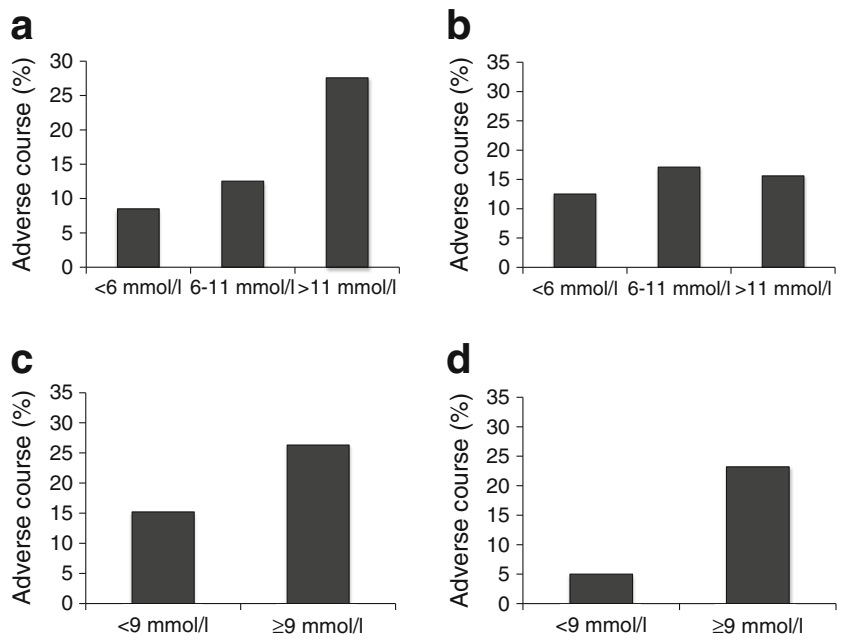

Fig. 1 Risk for 30 day adverse clinical course according to glucose level on admission (a) without diabetes $(p=0.014)$ and (b) with diabetes $(p=0.895)$, and $96 \mathrm{~h}$ mean glucose level (c) without diabetes $(p=0.204)$ and $(\mathbf{d})$ with diabetes $(p=0.015)$

observations, our study expands the evidence to patients with CAP. Our data show a strong association between increased glucose levels and adverse outcome, which is largely independent of pneumonia severity as measured by the CURB65 score.

In a previous study [15] involving sepsis patients hospitalised in medical wards, an association between increased blood glucose values over $72 \mathrm{~h}$ and higher mortality was found in nondiabetic patients. In the current report (using $96 \mathrm{~h}$ rather than $72 \mathrm{~h}$ values), this association was not significant. This lack of significance may be explained by the relatively low number of non-diabetic patients with increased initial blood glucose levels who were included in this analysis (i.e. by low statistical power). Still, prolonged hyperglycaemia over $96 \mathrm{~h}$ was associated with adverse outcomes in the diabetic population. This suggests that diabetic patients may tolerate sporadic hyperglycaemia better than non-diabetic patients, whereas prolonged hyperglycaemia increases the risk for treatment failure in the diabetic population.

Preclinical studies have linked hyperglycaemia to different physiological defects such as platelet malfunction, endothelial dysfunction, impaired chemotaxis, diminished granulocyte adherence, decreased phagocytosis and reduced microbicidal capacity (reviewed in [3]). In vitro studies have shown that
Table 2 Association between glucose range on admission and 30 day outcomes by diabetes status

\begin{tabular}{|c|c|c|c|c|}
\hline \multirow[t]{2}{*}{ Diabetes status/outcome } & \multicolumn{2}{|l|}{ Univariate } & \multicolumn{2}{|l|}{ Multivariate $^{\mathrm{a}}$} \\
\hline & OR $(95 \% \mathrm{CI})$ & $p$ value & OR $(95 \% \mathrm{CI})$ & $p$ value \\
\hline \multicolumn{5}{|l|}{ Non-diabetic patients } \\
\hline \multicolumn{5}{|c|}{ Adverse clinical course (ICU admission, all-cause death or both) } \\
\hline Euglycaemia $(<6.0 \mathrm{mmol} / \mathrm{l})$ & Reference & & Reference & \\
\hline Moderate hyperglycaemia (6-11 mmol/1) & $1.5(0.9,2.7)$ & 0.133 & $1.4(0.8,2.4)$ & 0.283 \\
\hline Severe hyperglycaemia (>11 mmol/l) & $4.1(1.6,10.6)$ & 0.004 & $3.0(1.1,8.0)$ & 0.032 \\
\hline \multicolumn{5}{|l|}{ ICU admission } \\
\hline Euglycaemia $(<6.0 \mathrm{mmol} / \mathrm{l})$ & Reference & & Reference & \\
\hline Moderate hyperglycaemia (6-11 mmol/l) & $1.9(1.0,3.9)$ & 0.064 & $1.8(0.9,3.6)$ & 0.116 \\
\hline Severe hyperglycaemia ( $>11 \mathrm{mmol} / \mathrm{l})$ & $4.0(1.2,12.6)$ & 0.019 & $3.0(0.9,9.6)$ & 0.072 \\
\hline \multicolumn{5}{|l|}{ Death } \\
\hline Euglycaemia $(<6.0 \mathrm{mmol} / \mathrm{l})$ & Reference & & Reference & \\
\hline Moderate hyperglycaemia (6-11 mmol/l) & $1.0(0.5,2.3)$ & 0.934 & $0.9(0.4,2.0)$ & 0.745 \\
\hline Severe hyperglycaemia (>11 mmol/l) & $4.4(1.4,14.3)$ & 0.013 & $2.9(0.8,9.9)$ & 0.090 \\
\hline \multicolumn{5}{|l|}{ Diabetic patients } \\
\hline \multicolumn{5}{|c|}{ Adverse clinical course (ICU admission, all-cause death or both) } \\
\hline Euglycaemia $(<6.0 \mathrm{mmol} / \mathrm{l})$ & Reference & & Reference & \\
\hline Moderate hyperglycaemia (6-11 mmol/1) & $1.4(0.3,7.1)$ & 0.45 & $1.6(0.3,8.0)$ & 0.55 \\
\hline Severe hyperglycaemia $(>11 \mathrm{mmol} / \mathrm{l})$ & $1.3(0.3,6.6)$ & 0.31 & $1.6(0.3,8.4)$ & 0.56 \\
\hline \multicolumn{5}{|l|}{ ICU admission } \\
\hline Euglycaemia $(<6.0 \mathrm{mmol} / \mathrm{l})$ & Reference & & Reference & \\
\hline Moderate hyperglycaemia (6-11 mmol/l) & $1.0(0.2,5.4)$ & 0.943 & $1.1(0.2,5.7)$ & 0.888 \\
\hline Severe hyperglycaemia $(>11 \mathrm{mmol} / \mathrm{l})$ & $0.9(0.2,4.6)$ & 0.860 & $1.0(0.2,5.5)$ & 0.996 \\
\hline \multicolumn{5}{|l|}{ Death } \\
\hline Euglycaemia $(<6.0 \mathrm{mmol} / \mathrm{l})$ & Reference & & Reference & \\
\hline Moderate hyperglycaemia (6-11 mmol/l) & $1.1(0.1,9.7)$ & 0.961 & $1.1(0.1,10.9)$ & 0.906 \\
\hline Severe hyperglycaemia (>11 mmol/l) & $0.7(0.1,7.6)$ & 0.798 & $0.9(0.1,9.9)$ & 0.951 \\
\hline
\end{tabular}


Fig. 2 Association between serum glucose level on admission and selected inflammation/ infection biomarkers during hospitalisation: CRP (a), WBC (c), PCT (e) and ProADM (g) in non-diabetic patients and CRP (b), WBC (d), PCT (f) and ProADM (h) in diabetic patients. Black lines, euglycaemia; grey lines, moderate hyperglycaemia; dashed lines, severe

hyperglycaemia. In non-diabetic patients, higher admission glucose ranges were significantly associated with higher initial and follow-up levels of CRP, WBC and ProADM for all days $(p<0.05$, ANOVA). No differences were found for PCT on any day ( $p \geq 0.05$, ANOVA). In the diabetic population, no significant differences were found for any marker on any day ( $p \geq 0.05$, ANOVA), except for CRP on the day of admission $(p<0.05$, ANOVA $)$
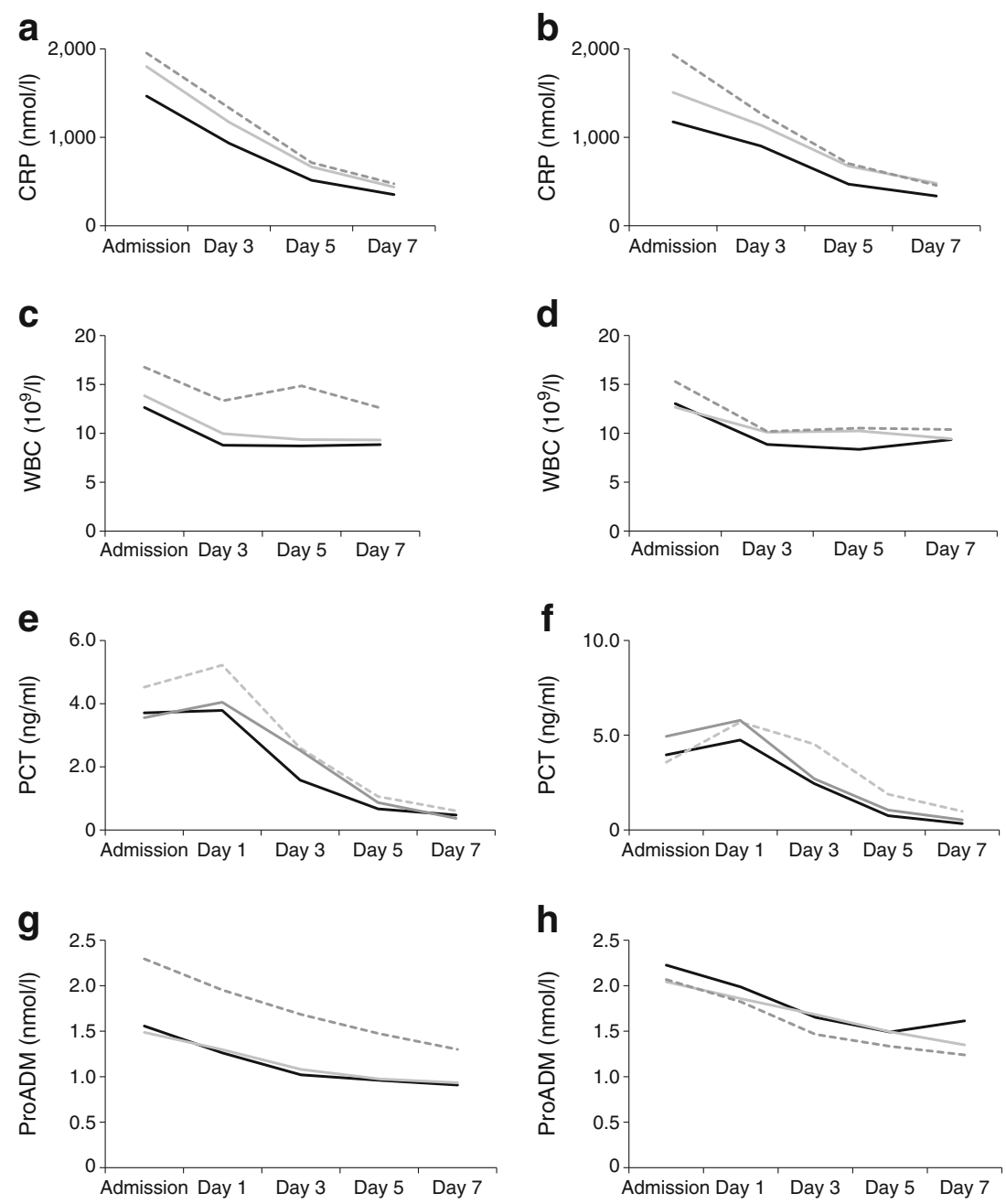

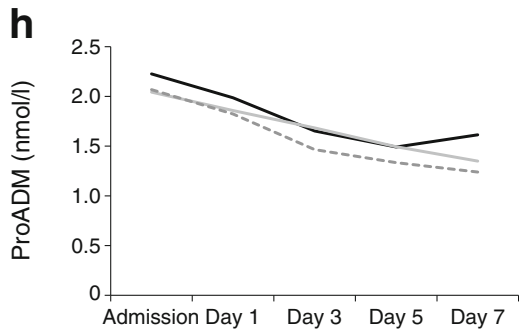

these effects are at least partly reversible when glucose is lowered to normal values. Within our analysis, we investigated the effects of hyperglycaemia on the kinetics of different blood biomarkers of inflammation and infection. Specifically, we investigated levels of CRP and WBC, two markers of inflammation and stress, and of PCT and ProADM, two

Table 3 Association of mean glucose during the first 96 inpatient hours and adverse clinical course (ICU admission, all-cause death or both) by diabetes status

\begin{tabular}{|c|c|c|c|c|}
\hline \multirow[t]{2}{*}{ Risk for adverse clinical course } & \multicolumn{2}{|l|}{ Univariate } & \multicolumn{2}{|l|}{ Multivariate $^{\mathrm{a}}$} \\
\hline & OR $(95 \% \mathrm{CI})$ & $p$ value & OR $(95 \% \mathrm{CI})$ & $p$ value \\
\hline \multicolumn{5}{|l|}{ Non-diabetic patients } \\
\hline Risk per $10 \%$ increase in $96 \mathrm{~h}$ mean glucose level (decile) & $1.1(0.9,1.4)$ & 0.395 & $1.1(0.9,1.4)$ & 0.514 \\
\hline \multicolumn{5}{|l|}{ Hyper- vs euglycaemia } \\
\hline Euglycaemia $(\leq 9.0 \mathrm{mmol} / \mathrm{l})$ & Reference & & Reference & \\
\hline Hyperglycaemia $(\geq 9.0 \mathrm{mmol} / \mathrm{l})$ & $2.0(0.5,7.3)$ & 0.3 & $1.8(0.4,7.2)$ & 0.431 \\
\hline \multicolumn{5}{|l|}{ Diabetic patients } \\
\hline Risk per $10 \%$ increase in $96 \mathrm{~h}$ mean glucose level (decile) & $1.4(1.1,1.8)$ & 0.015 & $1.4(1.1,1.9)$ & 0.014 \\
\hline \multicolumn{5}{|l|}{ Hyper- vs euglycaemia } \\
\hline Euglycaemia $(\leq 9.0 \mathrm{mmol} / \mathrm{l})$ & Reference & & Reference & \\
\hline Hyperglycaemia $(\geq 9.0 \mathrm{mmol} / \mathrm{l})$ & $5.7(1.2,27.1)$ & 0.027 & $5.4(1.1,25.8)$ & 0.033 \\
\hline
\end{tabular}

${ }^{\mathrm{a}}$ Adjusted for CURB65 
Fig. 3 Association between biomarkers and serum glucose during the first 96 inpatient hours. CRP (a), WBC (c), PCT (e) and ProADM (g) in non-diabetic patients and CRP (b), WBC (d), PCT (f) and ProADM (h) in diabetic patients. Black line, mean glucose $<9 \mathrm{mmol}$; dashed line, mean glucose $\geq 9 \mathrm{mmol} / \mathrm{l}$. In diabetic patients, significant differences were found for CRP on all days ( $p<0.05$, ANOVA), and for WBC on days 5 and 7 ( $p<0.05$, ANOVA). For nondiabetic patients, no significant differences were found for any marker on any day $(p>0.05$, ANOVA) a

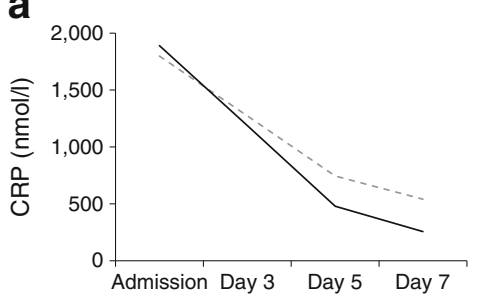

C

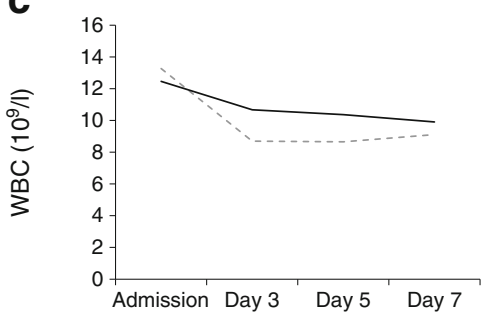

e
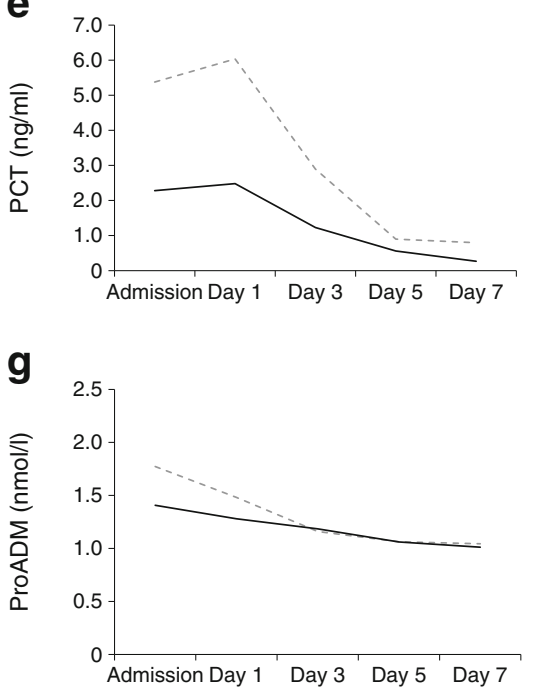

b

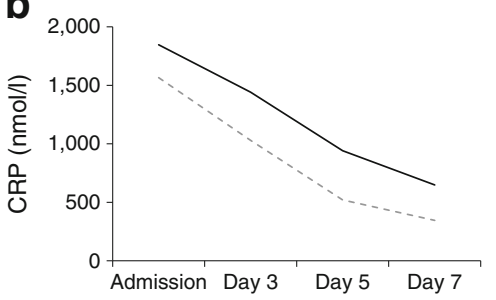

d

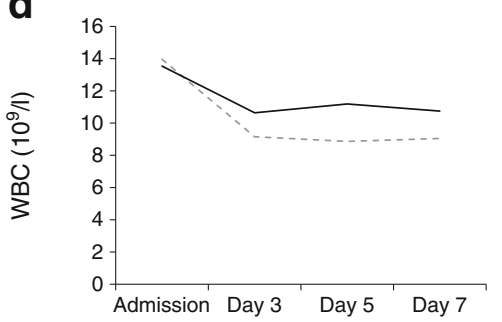

f

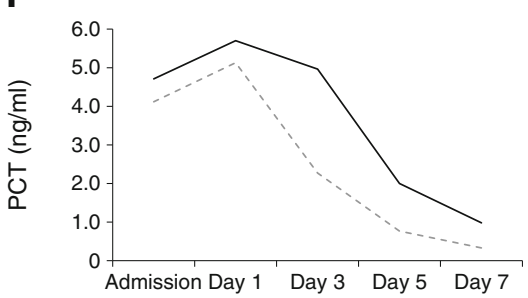

h

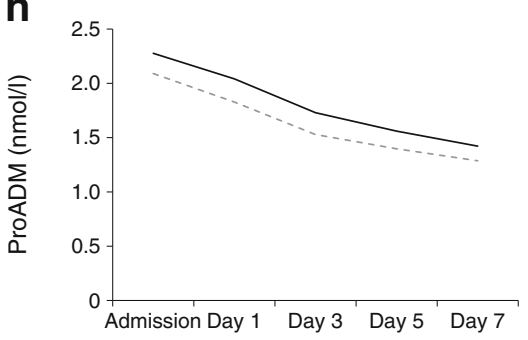

circulating substances referred to as 'hormokines', since they normally follow hormonal behaviour (i.e. expression in neuroendocrine cells and systemic action) but in response to inflammation or other physiological stress follow cytokine behaviour (i.e. expression in numerous cell types throughout the body and local action) [33]. To our knowledge, this is the first report linking hyperglycaemia to a more pronounced inflammatory response in non-diabetic individuals, as demonstrated by increased levels of CRP, WBC and ProADM over the first 7 days of inpatient treatment.

From our analysis, the question of association vs causation cannot conclusively be answered. Severe illness and systemic infection are associated with an increase in pro-inflammatory cytokines, such as IL-1, IL- 6 and TNF- $\alpha$ among others [34]. These cytokines trigger the expression of CRP, PCT, ProADM and $\mathrm{WBC}$ as part of the immune response [35-41]. Also, severe illness and systemic infection activate the sympathoadrenal system and the hypothalamic-pituitary-adrenal axis
[42-45], which causes peripheral insulin resistance, ultimately leading to hyperglycaemia [42]. To study the effects of hyperglycaemia on outcomes, we adjusted the analysis for CAP severity. Still, residual confounding is possible, and only an interventional study can definitively prove causation, were such a study to observe lower biomarker levels in patients treated with insulin compared with a control group.

In diabetic patients, no association between initial blood glucose level or blood glucose level over the first $96 \mathrm{~h}$ of hospitalisation and inflammatory biomarkers was found. This may be explained by the fact that insulin use in diabetic patients suppresses the production of nuclear factor kappa-light-chainenhancer of activated B cells, which modulates the expression of the pro-inflammatory cytokines, such as IL-1 and TNF- $\alpha$, ultimately leading to reduced blood levels of CRP, PCT, ProADM and leucocytes [46-48]. In our analysis, however, we did not find any evidence for effect modification of insulin treatment and biomarker levels. Yet, this observation may be due to the 
small sample and lack of standardisation regarding insulin treatment. Further, diabetic patients are frequently treated with statins, which have been associated with reduced inflammation [49]. Finally, it is possible that in diabetic individuals with chronic hyperglycaemia, acutely increased glucose levels do not cause the same toxic effects as in non-diabetic individuals.

The present study has several limitations. First, this is a secondary analysis of a prospective trial and is thus hypothesis-generating rather than definitive. There is a risk for selection bias because the most severely ill patients with possible imminent death may not have been included in ProHOSP, or may not have had initial blood glucose measurement due to their condition. There is also a risk for confounding by indication regarding the repeated glucose measurement, with more frequent measurements in patients with higher levels. We also assumed a linear trend between measurements, which may underestimate average glucose levels. Optimally, continuous glucose measurement would be used to assess the true glucose mean. Second, classification of diabetes status was based on self-report and information from medical charts. We were not able to gather data about diabetes severity, long-term glycaemic control (e.g. $\mathrm{HbA}_{1 \mathrm{c}}$ levels) or the time since diabetes diagnosis. Most importantly, our analysis shows associations but does not prove causation and thus it remains unclear whether correction of hyperglycaemia would reverse the observed effects on inflammatory response and clinical outcome. Finally, unlike other investigations, such as the RABBIT 2 surgery trial [50], we did not find any evidence for effect modification of insulin treatment on the association of glucose and clinical outcome, or biomarker levels. However, our sample may have been too small and lacked statistical power to detect such effects. Also, we were unable to compare the effect of different insulin regimens, as insulin regimens were up to the discretion of treating physicians and thus not standardised.

In conclusion, this analysis links initial hyperglycaemia in non-diabetic CAP patients, and prolonged hyperglycaemia in both diabetic and non-diabetic CAP patients, to a more pronounced inflammatory response and adverse CAP-related clinical outcome. We found no evidence that insulin treatment would affect these associations. Although critical-care trials have produced controversial data about optimal glucoselowering strategies, our data lend support to correcting hyperglycaemia - at least to levels of 9-10 $\mathrm{mmol} / \mathrm{l}$ or below. Yet, optimal glucose targets for insulin treatment of hyperglycaemia for diabetic and non-diabetic patients in the non-critical-care setting need to be better defined.

Acknowledgements We are grateful to the Data Safety and Monitoring Board, namely A. P. Perruchoud, S. Harbarth and A. Azzola, for continuous oversight of this trial and all local physicians, the nursing staff, the patients and their relatives who participated in this study. Especially, we thank the staff of the emergency room, medical clinics and central laboratories of the University Hospital Basel, the Cantonal Hospitals (Liestal, Aarau, Luzern and Muensterlingen) and the 'Buergerspital' Solothurn for their very helpful assistance, patience and technical support. We thank other members of the ProHOSP Study Group for their important help during the study (see electronic supplementary material [ESM] Study Group). We also thank R. J. Marlowe for editing the manuscript.

Funding This study was partly supported by a grant from the Swiss National Research Foundation (SNF 3200BO-116177/1) and also by contributions from Santésuisse and the Gottfried and Julia BangerterRhyner-Foundation, the University Hospital Basel, the Medical University Clinic Liestal, the Medical Clinic Buergerspital Solothurn, the Cantonal Hospitals Muensterlingen, Aarau and Lucerne, the Swiss Society for Internal Medicine (SGIM) and the Department of Endocrinology, Diabetology and Clinical Nutrition (University Hospital Basel).

Duality of interest PS, MCC and BM received support from Thermo Fisher Scientific (formerly $\mathrm{B} \cdot \mathrm{R} \bullet \mathrm{A} \cdot \mathrm{H} \bullet \mathrm{M} \bullet \mathrm{S} A G$ ). All other authors declare that there is no duality of interest associated with their contribution to this manuscript.

Contribution statement PS, NF, EG, AK and SH had the idea for and initiated the substudy, performed the statistical analyses and developed the initial draft of the manuscript. MC-C, RT, CHo, CHe, WZ and BM made substantial contributions to the conception and design of the initial trial and the acquisition of clinical data. All authors amended and commented on the manuscript, revising it critically for important intellectual content. All authors read and approved the final manuscript.

\section{References}

1. Delamaire M, Maugendre D, Moreno M, Le Goff MC, Allannic H, Genetet B (1997) Impaired leucocyte functions in diabetic patients. Diabet Med 14:29-34

2. Alexiewicz JM, Kumar D, Smogorzewski M, Klin M, Massry SG (1995) Polymorphonuclear leukocytes in non-insulin-dependent diabetes mellitus: abnormalities in metabolism and function. Ann Intern Med 123:919-924

3. Schuetz P, Castro P, Shapiro NI (2011) Diabetes and sepsis: preclinical findings and clinical relevance. Diabetes Care 34:771-778

4. Liberatore RR Jr, Barbosa SF, Alkimin M et al (2005) Is immunity in diabetic patients influencing the susceptibility to infections? Immunoglobulins, complement and phagocytic function in children and adolescents with type 1 diabetes mellitus. Pediatr Diabetes 6:206-212

5. Spatz M, Eibl N, Hink S et al (2003) Impaired primary immune response in type-1 diabetes. Functional impairment at the level of APCs and T cells. Cell Immunol 221:15-26

6. Edwards MS, Fuselier PA (1983) Enhanced susceptibility of mice with streptozotocin-induced diabetes to type II group B streptococcal infection. Infect Immun 39:580-585

7. Lepper PM, Ott S, Nuesch E et al (2012) Serum glucose levels for predicting death in patients admitted to hospital for community acquired pneumonia: prospective cohort study. BMJ 344:e3397

8. Esper AM, Moss M, Martin GS (2009) The effect of diabetes mellitus on organ dysfunction with sepsis: an epidemiological study. Crit Care 13:R18

9. Moss M, Guidot DM, Steinberg KP et al (2000) Diabetic patients have a decreased incidence of acute respiratory distress syndrome. Crit Care Med 28:2187-2192

10. Thomsen RW, Hundborg HH, Lervang HH, Johnsen SP, Sorensen HT, Schonheyder HC (2004) Diabetes and outcome of community- 
acquired pneumococcal bacteremia: a 10-year population-based cohort study. Diabetes Care 27:70-76

11. Graham BB, Keniston A, Gajic O, Trillo Alvarez CA, Medvedev S, Douglas IS (2010) Diabetes mellitus does not adversely affect outcomes from a critical illness. Crit Care Med 38:16-24

12. Van den Berghe G, Wilmer A, Hermans G et al (2006) Intensive insulin therapy in the medical ICU. N Engl J Med 354:449-461

13. van den Berghe G, Wouters P, Weekers F et al (2001) Intensive insulin therapy in critically ill patients. N Engl J Med 345:1359-1367

14. Investigators N-SS, Finfer S, Chittock DR et al (2009) Intensive versus conventional glucose control in critically ill patients. N Engl J Med 360:1283-1297

15. Schuetz P, Kennedy M, Lucas JM et al (2012) Initial management of septic patients with hyperglycemia in the noncritical care inpatient setting. Am J Med 125:670-678

16. Schuetz P, Christ-Crain M, Wolbers M et al (2007) Procalcitonin guided antibiotic therapy and hospitalization in patients with lower respiratory tract infections: a prospective, multicenter, randomized controlled trial. BMC Health Serv Res 7:102

17. Schuetz P, Christ-Crain M, Thomann R et al (2009) Effect of procalcitonin-based guidelines vs standard guidelines on antibiotic use in lower respiratory tract infections: the ProHOSP randomized controlled trial. JAMA 302:1059-1066

18. Schuetz P, Wolbers M, Christ-Crain M et al (2010) Prohormones for prediction of adverse medical outcome in community-acquired pneumonia and lower respiratory tract infections. Crit Care 14:R106

19. Schuetz P, Suter-Widmer I, Chaudri A et al (2011) Prognostic value of procalcitonin in community-acquired pneumonia. Eur Respir $\mathrm{J}$ 37:384-392

20. Renaud B, Schuetz P, Claessens YE, Labarere J, Albrich W, Mueller B (2012) Proadrenomedullin improves risk of early admission to ICU score for predicting early severe community-acquired pneumonia. Chest 142:1447-1454

21. Mimoz O, Benoist JF, Edouard AR, Assicot M, Bohuon C, Samii K (1998) Procalcitonin and C-reactive protein during the early posttraumatic systemic inflammatory response syndrome. Intensive Care Med 24:185-188

22. Guertler C, Wirz B, Christ-Crain M, Zimmerli W, Mueller B, Schuetz $P$ (2011) Inflammatory responses predict long-term mortality risk in community-acquired pneumonia. Eur Respir J 37:1439-1446

23. Umpierrez GE, Hellman R, Korytkowski MT et al (2012) Management of hyperglycemia in hospitalized patients in noncritical care setting: an endocrine society clinical practice guideline. J Clin Endocrinol Metab 97:16-38

24. Calverley PM, Walker P (2003) Chronic obstructive pulmonary disease. Lancet 362:1053-1061

25. Gonzales R, Sande MA (2000) Uncomplicated acute bronchitis. Ann Intern Med 133:981-991

26. Niederman MS, Mandell LA, Anzueto A et al (2001) Guidelines for the management of adults with community-acquired pneumonia. Diagnosis, assessment of severity, antimicrobial therapy, and prevention. Am J Respir Crit Care Med 163:1730-1754

27. Woodhead M, Blasi F, Ewig S et al (2005) Guidelines for the management of adult lower respiratory tract infections. Eur Respir J 26:1138-1180

28. Lim WS, van der Eerden MM, Laing R et al (2003) Defining community acquired pneumonia severity on presentation to hospital: an international derivation and validation study. Thorax 58:377-382

29. Kornum JB, Thomsen RW, Riis A, Lervang HH, Schonheyder HC, Sorensen HT (2007) Type 2 diabetes and pneumonia outcomes: a population-based cohort study. Diabetes Care 30:2251-2257

30. McAlister FA, Majumdar SR, Blitz S, Rowe BH, Romney J, Marrie TJ (2005) The relation between hyperglycemia and outcomes in 2, 471 patients admitted to the hospital with community-acquired pneumonia. Diabetes Care 28:810-815
31. Moghissi ES, Korytkowski MT, DiNardo M et al (2009) American Association of Clinical Endocrinologists and American Diabetes Association consensus statement on inpatient glycemic control. Diabetes Care 32:1119-1131

32. Stegenga ME, Vincent JL, Vail GM et al (2010) Diabetes does not alter mortality or hemostatic and inflammatory responses in patients with severe sepsis. Crit Care Med 38:539-545

33. Christ-Crain M, Muller B (2007) Biomarkers in respiratory tract infections: diagnostic guides to antibiotic prescription, prognostic markers and mediators. Eur Respir J 30:556-573

34. Cerami A (1992) Inflammatory cytokines. Clin Immunol Immunopathol 62:S3-S10

35. Hofbauer KH, Schoof E, Kurtz A, Sandner P (2002) Inflammatory cytokines stimulate adrenomedullin expression through nitric oxidedependent and -independent pathways. Hypertension 39:161-167

36. Moshage HJ, Roelofs HM, van Pelt JF et al (1988) The effect of interleukin-1, interleukin- 6 and its interrelationship on the synthesis of serum amyloid A and C-reactive protein in primary cultures of adult human hepatocytes. Biochem Biophys Res Commun 155:112117

37. Redl H, Schlag G, Togel E, Assicot M, Bohuon C (2000) Procalcitonin release patterns in a baboon model of trauma and sepsis: relationship to cytokines and neopterin. Crit Care Med 28:3659-3663

38. Redl H, Schiesser A, Togel E, Assicot M, Bohuon C (2001) Possible role of TNF on procalcitonin release in a baboon model of sepsis. Shock 16:25-27

39. Ulich TR, del Castillo J, Keys M, Granger GA (1987) Recombinant human alpha lymphotoxin (tumor necrosis factor-beta) induces peripheral neutrophilia and lymphopenia in the rat. Am J Pathol 128:512

40. Ulich TR, del Castillo J, Keys M, Granger GA, Ni RX (1987) Kinetics and mechanisms of recombinant human interleukin 1 and tumor necrosis factor-alpha-induced changes in circulating numbers of neutrophils and lymphocytes. J Immunol 139:3406-3415

41. Ulich TR, del Castillo J, Guo KZ (1989) In vivo hematologic effects of recombinant interleukin- 6 on hematopoiesis and circulating numbers of RBCs and WBCs. Blood 73:108-110

42. Dungan KM, Braithwaite SS, Preiser JC (2009) Stress hyperglycaemia. Lancet 373:1798-1807

43. Ishihara M, Kagawa E, Inoue I et al (2007) Impact of admission hyperglycemia and diabetes mellitus on short- and long-term mortality after acute myocardial infarction in the coronary intervention era. Am J Cardiol 99:1674-1679

44. Andrews RC, Walker BR (1999) Glucocorticoids and insulin resistance: old hormones, new targets. Clin Sci (Lond) 96:513-523

45. Gelfand RA, Matthews DE, Bier DM, Sherwin RS (1984) Role of counterregulatory hormones in the catabolic response to stress. J Clin Invest 74:2238-2248

46. Dandona P, Aljada A, Mohanty P et al (2001) Insulin inhibits intranuclear nuclear factor kappaB and stimulates IkappaB in mononuclear cells in obese subjects: evidence for an anti-inflammatory effect? J Clin Endocrinol Metab 86:3257-3265

47. Das UN (2001) Is insulin an antiinflammatory molecule? Nutrition 17:409-413

48. Garg R, Chaudhuri A, Munschauer F, Dandona P (2006) Hyperglycemia, insulin, and acute ischemic stroke: a mechanistic justification for a trial of insulin infusion therapy. Stroke 37:267273

49. Donnino MW, Cocchi MN, Howell M et al (2009) Statin therapy is associated with decreased mortality in patients with infection. Acad Emerg Med 16:230-234

50. Umpierrez GE, Smiley D, Jacobs S et al (2011) Randomized study of basal-bolus insulin therapy in the inpatient management of patients with type 2 diabetes undergoing general surgery (RABBIT 2 surgery). Diabetes Care 34:256-261 